\title{
Light-driven dynamic adhesion on photo-sensitized nematic liquid crystalline elastomers
}

Takuya Ohzono, ${ }^{* a}$ Yasuo Norikane, ${ }^{a}$ Mohand O. Saed,${ }^{b}$ and Eugene M. Terentjev ${ }^{* b}$

a. Research Institute for Electronics and Photonics, National Institute of Advanced Industrial Science and Technology (AIST) 1-1-1 Higashi, Tsukuba 305-8565, Japan.

b. Cavendish Laboratory, University of Cambridge, J.J. Thomson Avenue, Cambridge, CB3 0HE, United Kingdom.

KEYWORDS: nematic liquid crystal elastomer, adhesion, soft elasticity, photo-thermal effect, robot hands, phase transition

ABSTRACT: In liquid crystal elastomers (LCE), the internal mechanical loss increases around the nematic-isotropic phase transition, and remains high all through the nematic phase, originating from the internal orientational relaxation related to the so-called 'soft elasticity'. Since the viscoelastic dissipation of the materials affects their adhesion properties, the nematicisotropic phase transition can cause dramatic changes in the adhesion strength. Although the phase transitions can generally be induced by heat, here we demonstrate the light-driven transition in dynamic adhesion in dye-doped nematic LCE. The special dye is chosen to efficiently generate local heat on light absorption. The adhesion strength is lowered with fine 
tunability depending on the light power, which governs the effective local temperature, and through that the viscoelastic damping of the system. We demonstrate the light-assisted dynamic control of adhesion in a $90^{\circ}$-peel test, and in pick-and-release of objects, which may lead to the development of stimuli-responsive adhesive systems with fine spatio-temporal controls.

\section{INTRODUCTION}

Pressure sensitive adhesives (PSA) ${ }^{1,2}$ form a class of applications of viscoelastic elastomers and gels, which generally provide quick adhesion after applying light pressure. These materials have entered commercial use in applications such as tapes and transient fixation glues. Since most of PSAs are not intended to perform permanent adhesion, the ability of detachment on demand is also important. Intrinsically, the characteristics of adhesion and detachment are a trade-off: the larger peeling force is required to detach the PSA from the adhered counter surface with the higher adhesion. Thus, if we could reduce the peeling force at the desired moment and location, the cycle of strong adhesion and easy detachment becomes much more efficient. For this purpose, the materials that can change the adhesion strength upon external triggers, e.g., temperature, chemicals, electrical and mechanical fields, would be required. However, they were limited to some stimuli-responsive polymers ${ }^{3}$ and their combinations to surfaces with transformable topography in the celebrated 'gecko feet' effect ${ }^{4-8}$.

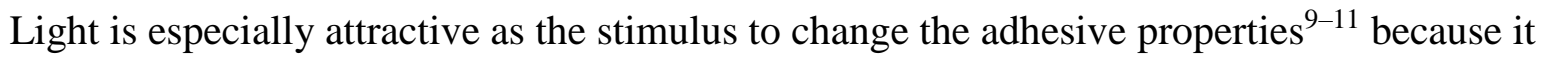
allows the fine spatio-temporal control remotely, without direct contact with the system and electric wiring to the adhesive interface. By incorporating the photosensitive units that directly or indirectly affect the adhesive properties such as liquid/solid phases, and their viscoelastic 
characteristics and shapes, various materials systems with reversible or irreversible switching of adhesive response have been proposed ${ }^{10}$.

For some applications, such as robot gripping hands and the temporary fixation in semiconductor manufacturing, quick reversibility of the adhesives regarding both chemistry and macroscopic shapes without leaving residues on the counter objects becomes especially important. For this purpose, adhesive systems based on soft crosslinked elastomers with their modulus well below 1 MPa without additive tackifires (low-molecular weight compounds often used in PSAs for increasing stickiness) are preferred ${ }^{1,2}$. As for the key mechanism of the stimuli-response of the elastomers, the phase transitions are suitable, because the adhesion is modulated via the associated mechanical properties while keeping the film from flowing. Especially, the transitions with the well-defined and controllable transition points, which are often accompanied by the liquid crystal phases ${ }^{12}$, are favored.

Nematic liquid crystal elastomers (LCE) $)^{13}$ are a promising group of such materials, since their moduli are typically less than $1 \mathrm{MPa}$, and their viscoelastic damping is dramatically affected by the internal rotations of nematic director, in one of manifestations of the dynamic 'soft elasticity' effect $^{14-16}$. Moreover, upon heating, nematic LCE exhibit the phase transition into the isotropic state, which is an ordinary elastomer with a much lower viscoelasticity damping than LCE. Since the strength of adhesion is closely related to the viscoelastic damping ${ }^{1,2,17,18}$, it has been demonstrated that tangible change of adhesion occurs on crossing the nematic-isotropic transition in $\mathrm{LCE}^{19}$.

Although the underlying reason for changing the nematic order parameter is thermal, there have been several photosensitized systems developed in LCE, mainly to achieve the mechanical 
actuation $^{20}$. The response of the internal nematic order to light has been achieved with the use of azobenzene units $^{21-23}$, carbon nanotubes ${ }^{24}$, gold nanorods ${ }^{25}$, conjugated polymers ${ }^{26}$, as well as

specialized dyes ${ }^{22,27,28}$. However, the parallel effect of light-induced change in adhesion through manipulation of viscoelastic dissipation upon the phase transition has not been explored yet.

Here we demonstrate the dynamic adhesion controlled by white light in as-prepared polydomain nematic LCE doped with an infrared dye as the photosensitizer responding to the longer wavelength parts of the illumination spectrum. The heat generated by light absorption induces nematic-isotropic phase transition, locally if the illuminated spot is small. As a result, the decreased viscoelastic damping in the isotropic phase causes a dramatic reduction in adhesive strength, leading to detachment from the counter surface. The relationship between the light power, dye-concentration, and adhesion is investigated. The spatio-temporal control of adhesion is also demonstrated via the localized and intermittent illumination.

\section{RESULTS AND DISCUSSIONS}

General characterization. Having tested a variety of photosensitizers, we focus on the wellknown infrared dye indocyanine green (ICG), which is used in a range of technologies: from laser welding of polymers to medical therapy. The components that produce our ICG-doped LCE are shown in Fig. 1a. The films show strong absorption in near-infra red (NIR) region of spectrum, and only weakly absorbs deep blue light (Fig. 1b), resulting in the greenish appearance (Fig. 1c). At the wavelength of $800 \mathrm{~nm}$, absorbance of the ICG-doped LCE at $c=0.2 \mathrm{wt} \%$ is $\sim 250$ times as large as that without dye. The calorimetry studies suggest that the nematic phase exists between the glass transition at $T_{\mathrm{g}}$, which was difficult to determine precisely, but certainly 
below $-30^{\circ} \mathrm{C}$, and the isotropic transition around $T_{\mathrm{NI}} \sim 60^{\circ} \mathrm{C}$ (Fig. 1d). The as-prepared LCE film in the nematic phase is opaque due to the strong light scattering from the randomly mis-aligned nematic domains ${ }^{13}$. Upon heating above $T_{\mathrm{NI}}$, the film becomes transparent as the birefringence contrast between misaligned nematic domains is reduced in the isotropic state, as in ordinary homogeneous elastomers, further confirming the uniform distribution of dye molecules in the LCE films. 
(a) Mesogens

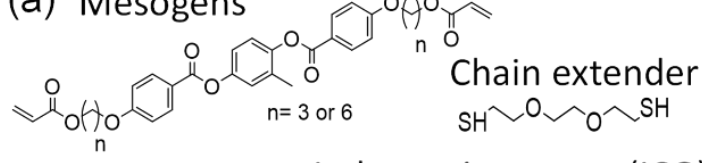

Crosslinker

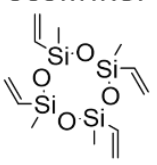

(b)
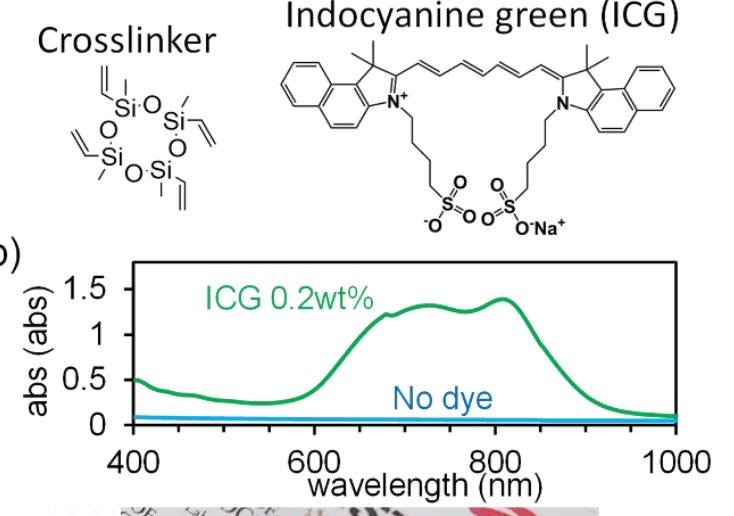

(c)

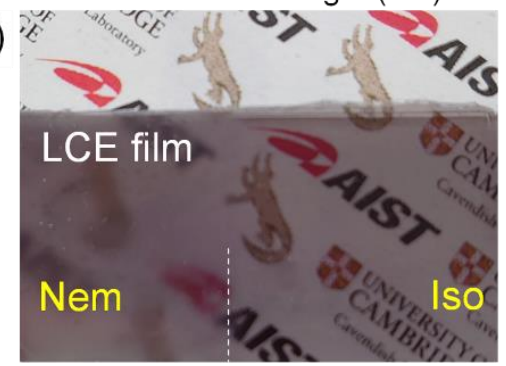

(d)

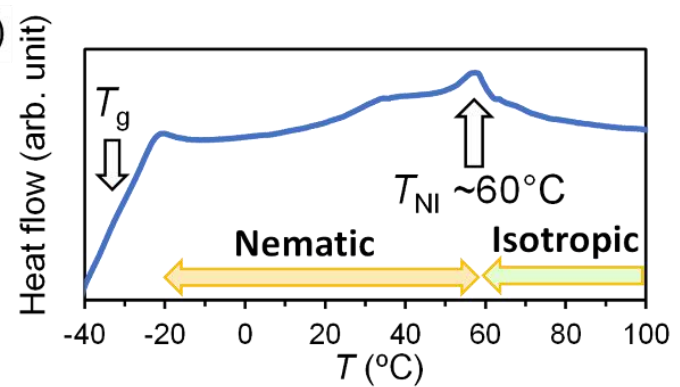

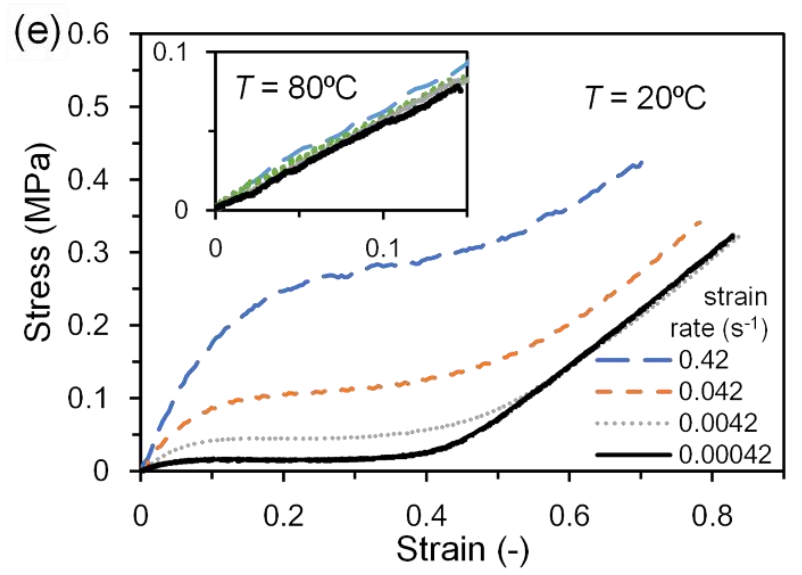

Figure 1. (a) Chemical structures of monomers for the main-chain LCE, and the ICG dye added as the photosensitizer. (b) Light absorption spectra of ICG-doped at $c=0.2 \mathrm{wt} \%$ and non-doped LCE films. (c) Visual appearance of LCE film (ICG-doped at $c=0.2 \mathrm{wt} \%$ ). Note the phase-dependent transparency. (d) DSC curve on heating, showing the phase transition temperatures. (e) Stress-strain curves at $T=20^{\circ} \mathrm{C}$ (nematic) and $80^{\circ} \mathrm{C}$ (isotropic) at different strain rates. Note, the little dependence of stress-strain response on the strain rate in isotropic phase (inset). No noticeable change on the stress-strain response was found on LCEs with different dye content. 


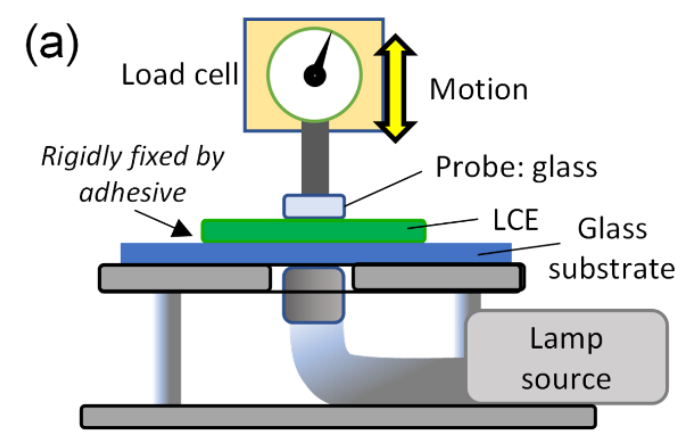

(b)
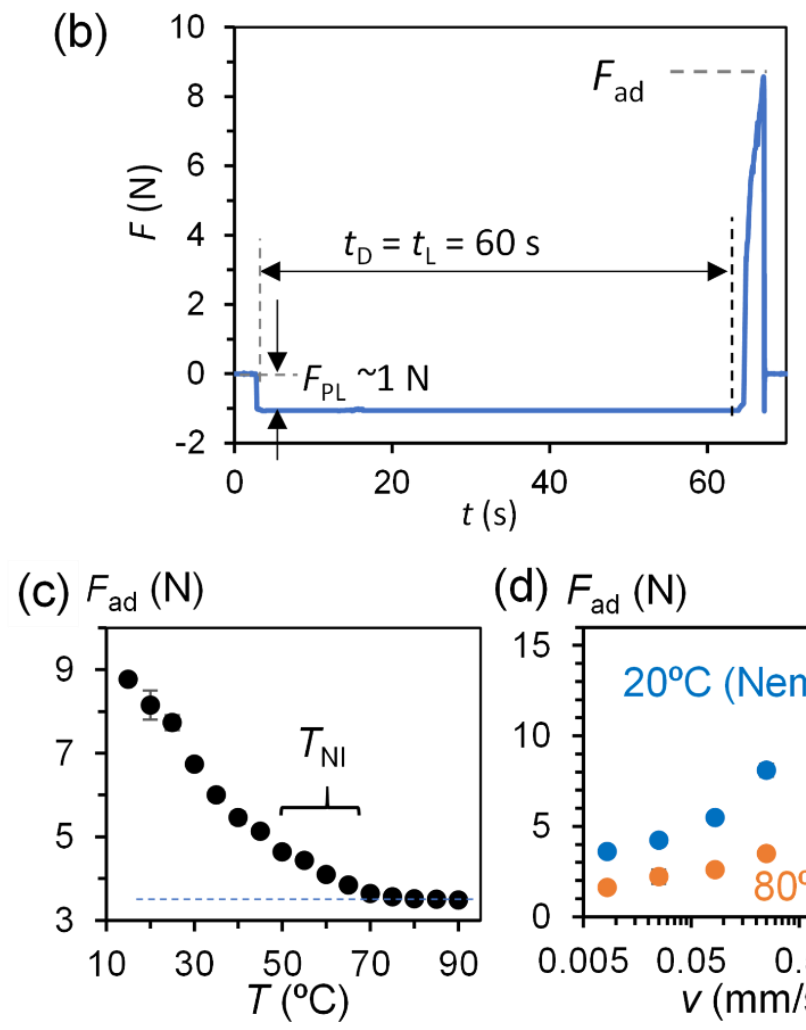

(d) $F_{\text {ad }}(\mathrm{N})$

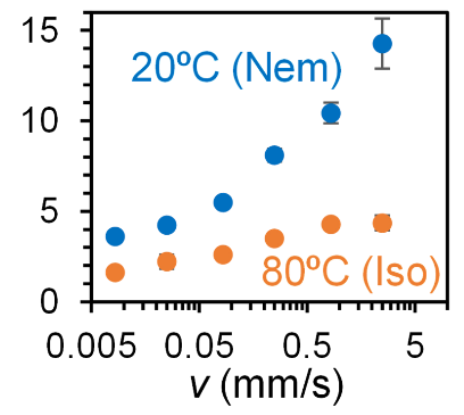

Figure 2. (a) Experimental setup for evaluation of adhesion strength under light irradiation. (b) Typical force curve in the adhesion experiment. The dwell time, $t_{\mathrm{D}}$, the light exposure time, $t_{\mathrm{L}}$, the pre-load, $L_{\mathrm{P}}$, and adhesion force, $F_{\text {ad }}$, are labelled in the plot. (c) Temperature-dependent adhesion force, $F_{\text {ad }}(T)$, at pulling speed $v=0.25 \mathrm{~mm} / \mathrm{s}$ without light irradiation. (d) Velocity-dependent adhesion force, $F_{\text {ad }}(v)$, at $T=20^{\circ} \mathrm{C}$ and $80^{\circ} \mathrm{C}$ without light irradiation. 
Figure 1e (inset) indicates that the LCE in the isotropic state shows the ordinary linear stressstrain relation and with little dependency on strain rate. This simply reflects the fact that the dynamic-mechanical response of the isotropic elastomer has the rubber plateau modulus up to the frequencies of $1 \mathrm{~Hz}^{29}$. In contrast, the stress-strain response in the nematic phase shows the typical soft elasticity ${ }^{13,14,30}$, in this case reflecting the re-orientation of the director during polydomain-monodomain transition. The corresponding dynamic-mechanical response of nematic LCE is well-studied, showing the dramatic increase of the loss factor (tan $\delta$ ) across the whole range of the nematic phase ${ }^{13-16}$.

Adhesion properties without light irradiation. Typical experiments to measure adhesion strength were performed with the setup shown in Fig. 2a and produce the force response illustrated in Fig. 2b. Firstly, we discuss the underlying temperature-dependent adhesion without light, as shown in Figs. 2c and 2d. The adhesion force, $F_{\text {ad }}$ (as defined in Fig. 2a) decreases on heating, as expected from the previous report on a similar main-chain LCE system ${ }^{19}$. The observed higher adhesion in the nematic phase is mainly due to the high loss factor, in contrast to the isotropic elastomer state, where no independent nematic director relaxation is present, and $\tan \delta$ is low.

Unfortunately, the detailed relationship between the actual energy dissipation upon de-bonding and the viscoelastic properties of the material, e.g., frequency-dependent storage and loss moduli, is still difficult to discuss here due to the very complex time-dependent deformation at the peeling front ${ }^{2}$. Nevertheless, this mechanism may directly manifest in the adhesion dependence on pulling-speed, $v$. Indeed, the LCE film shows the increase of adhesion with $v$, supporting the strong correlation between adhesion and viscoelastic damping indicated 
qualitatively by the rate-dependent mechanical response seen in Fig. 1e. Moreover, the increase rate in the nematic phase $\left(20^{\circ} \mathrm{C}\right)$ is clearly larger than that of the isotropic phase $\left(80^{\circ} \mathrm{C}\right)$, illustrating that the $T$-dependent loss modulus mainly governs the present adhesion strength, in accordance with the previous study ${ }^{19}$.

(a)
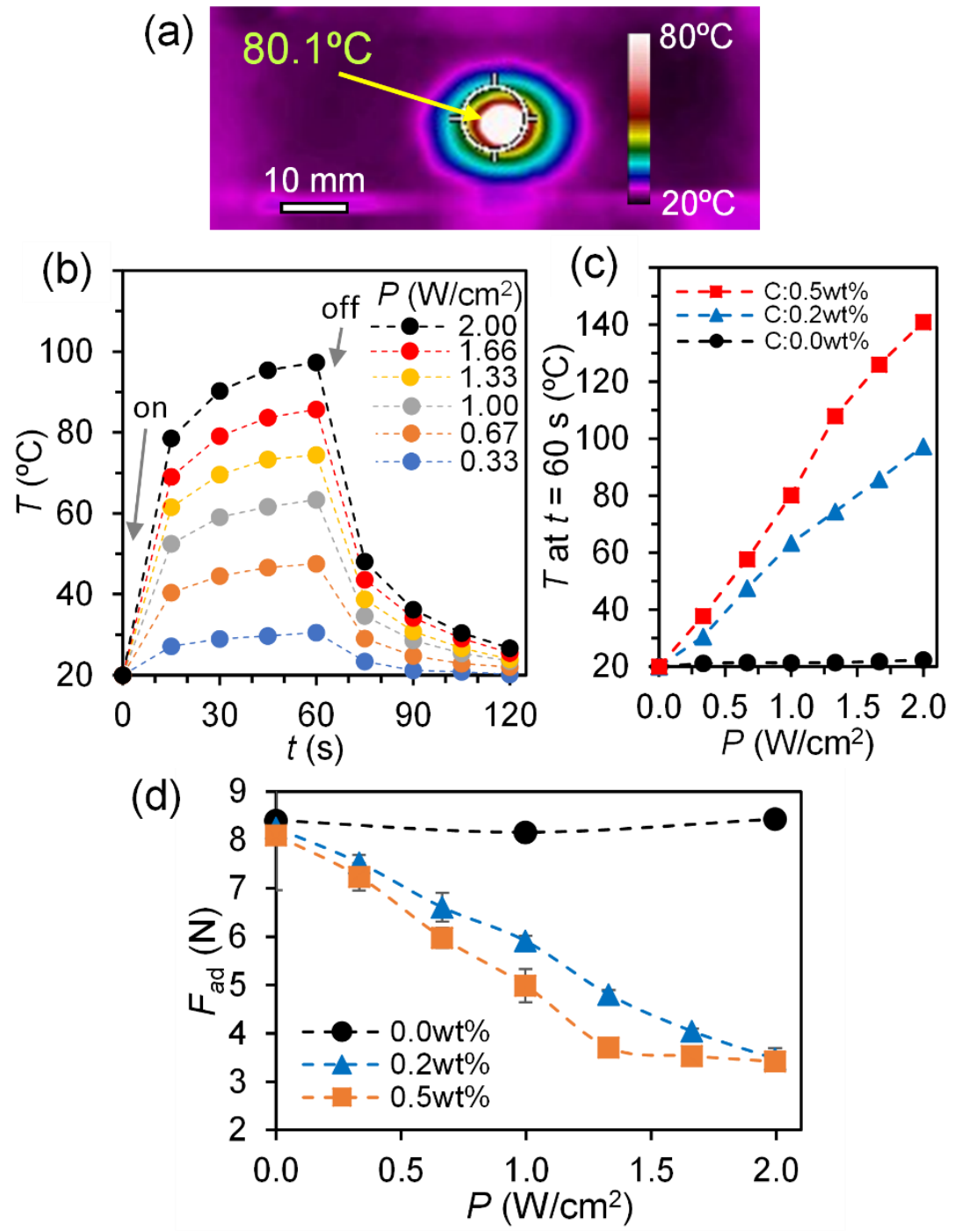

Figure 3. (a) Temperature distribution on LCE surface around the light spot, illuminated at $P=1 \mathrm{~W} / \mathrm{cm}^{2}$ for $t=60 \mathrm{~s}$ (ICG loading $c=0.5 \mathrm{wt} \%$ ). (b) The increase of local temperature with time on the LCE surface (ICG loading $c=0.2 \mathrm{wt} \%$ ). (c) Plots of $T$ at $t=60 \mathrm{~s}$ vs. illumination intensity $P$ on LCEs with different values of ICG doping. (d) Plots of adhesion force $F_{\text {ad }}$ Vs. $P$ at different ICG doping. $t_{\mathrm{D}}=t_{\mathrm{L}}=60 \mathrm{~s}, L_{\mathrm{P}}=1 \mathrm{~N}$ and $v=0.25 \mathrm{~mm} / \mathrm{s}$. 
Characterization of photothermal effect. To understand the light-induced dynamic adhesion, we need to characterize the relation between light irradiation and local temperature, to find the proper operation condition. Figure 3a shows a typical distribution of temperature across the film surface, around the location of the spotlight from the back side of the LCE film through a transparent glass slide, see Fig. 2a. Note that the top surface is exposed to ambient air for the measurement using a thermal imaging camera. Upon exposure to light, the temperature at the opposite sample surface evolves with time, $t$, depending on the light power, $P$, as shown in Fig. 3b. Within several seconds, the temperature quickly raises, and would generally approach a saturation value depending on various conditions, such as $P$, the ICG dye concentration, $c$, the ambient temperature, the sample thickness, $d$, and the thermal exchange coefficients with air and the solid glass support, into which the applied heat diffuses. The observed curves (Fig. 3b) clearly follow this trend within $60 \mathrm{~s}$. After the end of irradiation, the temperature dissipates with a similar time constant.

Photo-induced dynamic adhesion. The plots of temperature near its saturation level (at $t=60 \mathrm{~s}$ ) shown in Fig. $3 c$ confirm the expected effects of $P$ and $c$. It is possible, for photo-control of dynamic adhesion, to raise the local temperature towards, and beyond the present $T_{\mathrm{NI}} \sim 60^{\circ} \mathrm{C}$ by adjusting $P$ and/or $c$. The corresponding adhesion strength data are shown in Fig. $3 d$. Without the dye $(c=0 \mathrm{wt} \%)$, the high adhesion remains, suggesting that the system keeps the highly dissipative nematic state. In contrast, the dye-doped LCE the adhesion strength $F_{\text {ad }}$ shows a gradual decrease with $P$, demonstrating the fine tunability of adhesion by the light power.

The LCE with a higher dye concentration $(c=0.5 \mathrm{wt} \%)$ shows leveling of $F_{\text {ad }}$ at the higher range of $P$ above $\sim 1.3 \mathrm{~W} / \mathrm{cm}^{2}$. The result qualitatively corresponds to that observed under thermal control (Fig. 2c), which also has the plateau at the higher temperature of $70-90^{\circ} \mathrm{C}$. This suggests 
that the effective temperature of the LCE surface in adhesive contact with the glass probe falls into this range. Comparing this range of temperature to that measured without the glass probe, which was $110-140^{\circ} \mathrm{C}$ for the corresponding conditions $\left(P=1.3-2.0 \mathrm{~W} / \mathrm{cm}^{2}\right.$ and $\left.c=0.5 \mathrm{wt} \%\right)$ as shown in Fig. $3 \mathrm{c}, \sim 35^{\circ} \mathrm{C}$ drop is estimated. Since the solid glass probe absorbs more heat produced within the LCE film in contact, such a decrease in $T$ is reasonable. This also generally suggests that the photo-controlled adhesion may be greatly affected by the photothermal properties of the counter material to be adhered, e.g. its color and thermal conductivity. Nevertheless, by adjusting $P$ and photothermal energy conversion efficiency via the choice and concentration of the dye, flexible adaptation to various adhesive situations would be possible.

Spatio-temporal control of adhesion by light. One of the major merits of using light is the ease to specify the location of adhesion control. To demonstrate this, the $90^{\circ}$-peel test under spatiotemporal light irradiation is performed (Fig. 4a-4c and SI-Movie 1). Tangibly accelerated peeling only at the irradiated part is obtained.

Another system shown in Fig. $4 \mathrm{~d}$ and SI-Movie 2 demonstrates the pick-and-release of plastic balls, which would be a basic process in general production lines with robotic carriers. Without light, the surface of the LCE pad is sufficiently sticky to hold a PS ball. The detachment of the ball is induced by local light irradiation. In this case, both adherence and detachment finish within a few seconds (see SI-Movie 2) and the process of adherence and detachment can be repeated over 100 cycles without any noticeable change of the surface topography. The objects, which are transferable by this system, should depend on the true area of the contact, their photothermal properties, the weight, and the basic dynamic range of the adhesion of the used LCE as shown in Fig. 2c and 3d. However, in robotic gripping hands, the design could be more complicated (not relying on plain adhesion against gravity), and therefore the practical utility of 
the dynamically switching adhesion is much broader. The properly optimized systems would readily be applicable in many applications dealing with relatively small solid parts with smooth surfaces. Further tuning of LCE regarding the photosensitivity, the phase transition nature, and the viscoelasticity is possible via minor chemical modifications, which would further boost the broad applicability of the present concept of LCE-based light-controlled dynamic adhesion. 

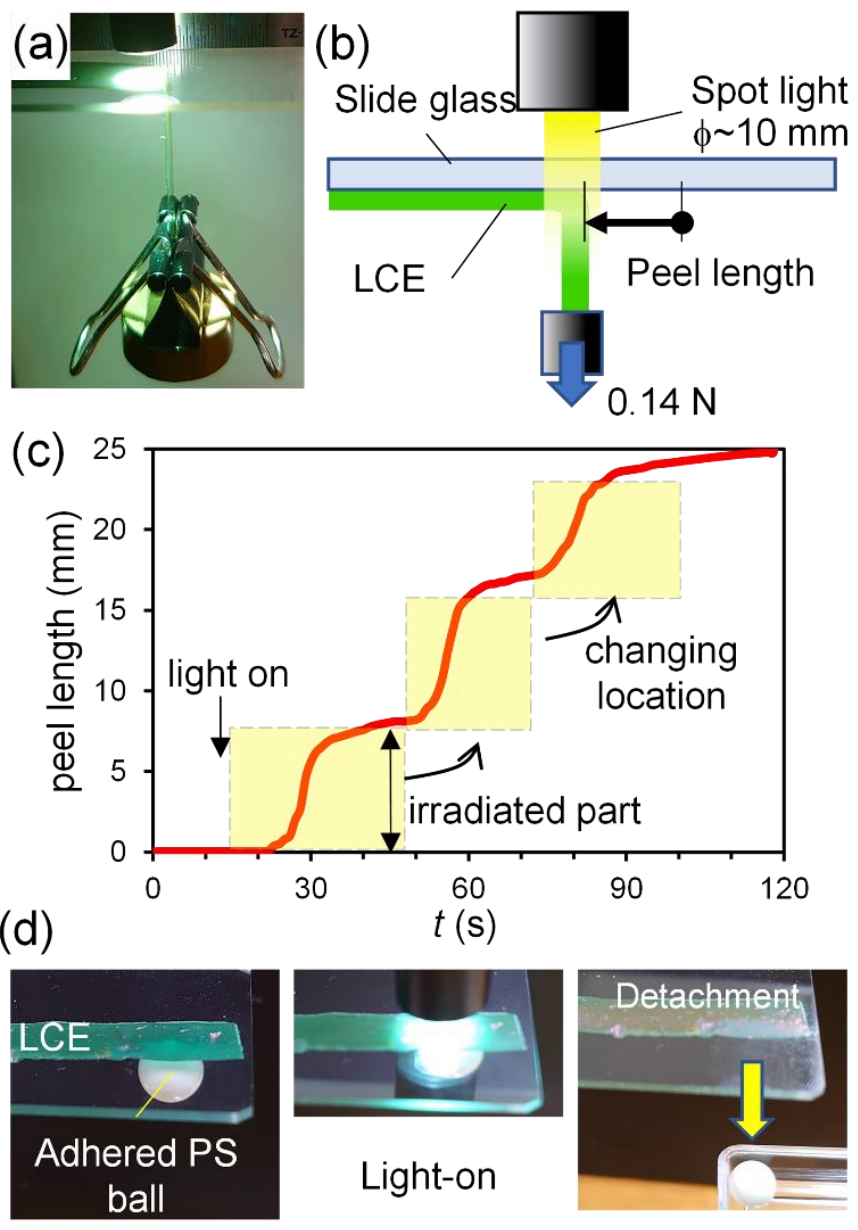

Figure 4. Demonstration of light-induced detachment. (a) An image during the experiment in the $90^{\circ}$-peel test configuration. Light is irradiated at the interface of the LCE strip adhered on the transparent glass slide, with the constant load of $14 \mathrm{~g}$ applied (giving the tension of $\sim 20 \mathrm{~N} / \mathrm{m}$ at the peeling edge). (b) Schematic side view of the $90^{\circ}$-peel experiment. (c) The change of the peeled length with time, $t$. Soon after light irradiation, only the part exposed to the light gets detached: see SI-Movie 1 for the process. (d) Sequential images of adherence of polystyrene (PS) ball (diameter of $5 \mathrm{~mm}$ and weight of $0.2 \mathrm{~g}$ ) and the light-induced detachment. Here, $c=0.2 \mathrm{wt} \%$ and $P \sim 1.4 \mathrm{~W} / \mathrm{cm}^{2}$. See SIMovie 2 for the process.

\section{CONCLUSIONS}

In conclusion, the light-controlled dynamic adhesion system is demonstrated, exploiting the high viscoelastic dissipation of nematic LCE and its photo-induced switching via doped photosensitizer. Other nematic LCE, including photosensitive units directly in the molecular 
structure, or using other dyes and nanoparticle doping may provide the same, or even better functionality. The combination of light responsive LCE and the additive manufacturing may provide more convenient systems applicable to handling tools, the general automatic production lines for electronic boards, micromechanical devices, smart tactile surfaces, and even beyond.

\section{EXPERIMENTAL SECTION}

Materials. LCE films were prepared using monomers shown in Fig. 1a. The indocyanine green dye $(\mathrm{ICG})^{31}$ was added at different concentrations as the light sensitizer mainly in the nearinfrared region. For preparation of LCE, we used the methods reported previously ${ }^{32-34}$, which include two-step crosslinking reactions of thiol-vinyl and thiol-acrylate additions, with some modifications. We followed a two-step thiol-ene/thiol-acrylate reaction sequence to prepare the light responsive LCE from commercially available starting materials. Acrylate mesogenic monomers, RM82 and RM257, was purchased from Wilshire Technologies, Inc. Thiol chain extender, 2,2'-(ethylenedioxy) diethanethiol (EDDT) and vinyl siloxane crosslinker, 2,4,6,8Tetramethyl-2,4,6,8-tetravinyl cyclotetrasiloxane (TMTVCTS), and catalysts triethylamine (TEA), were purchased from Sigma-Aldrich. The photoinitiator, Irgacure I-651 and the ICG dye, and the solvent dimethylformamide (DMF), were also purchased from Sigma-Aldrich, and were used as received.

The standard reaction scheme has been modified to accommodate the addition of the light responsive dye. We first prepared the thiol-terminated crosslinker via self-limiting thiol-ene click reaction between the initial tetra-vinyl crosslinker, TMTVCTS, and an isotropic dithiol, EDDT. The thiol-ene reaction was initiated via I651 (Photo-initiator, 1.5wt\%) and then radically 
crosslinked with $365 \mathrm{~nm}$ UV light for 60 minutes. In another container, ICG $(0,0.2$, or $0.5 \mathrm{wt} \%$ to the projected weight of the elastomer) was dissolved in DMF (40wt\%) and mixed with RM82/RM257 monomer mixture (80wt\% RM82 and 20wt\% RM257), selected to produce the nematic phase, rather than smectic. The mixture was then added into the to the thiol-terminated crosslinker. The mixture was dissolved by gentle heating $\left(80^{\circ} \mathrm{C}\right.$ for $\left.5 \mathrm{~min}\right)$ and stirring before adding TEA $(1 \mathrm{wt} \%)$ to catalyze the thiol-acrylate reaction. The solution was mixed rigorously, then degassed using a vacuum chamber, and then quickly transferred into a mold (two glass sides with $\sim 300 \mu \mathrm{m}$ spacer, coated with Rain-X anti-sticking agent). The reacting mixture was kept at $50^{\circ} \mathrm{C}$ to be fully crosslinked via the Michael addition reaction for $12 \mathrm{~h}$. The molar ratio used was kept to a fixed proportion of (acrylate : thiol : vinyl $)=1.0: 1.2: 0.2$. After polymerization, the samples were removed from the mold and placed in a vacuum oven at $80^{\circ} \mathrm{C}$ for $12 \mathrm{~h}$ to remove all solvent, resulting in the final thickness $d$ of $\sim 0.2 \mathrm{~mm}$.

Spectroscopy. Light absorption was characterized using a spectrophotometer (V-670, JASCO) on the slightly stretched ICG-doped LCE $(0.2 \mathrm{wt} \%)$ and non-doped one with the strain of $\sim 0.3$ to reduce the visible light scattering from the polydomain state ${ }^{13}$.

Thermal analysis. The nematic-isotropic phase transition temperature, $T_{\mathrm{NI}}$, of present LCEs were characterized using dynamic scanning calorimetry (DSC, DSC4000 PerkinElmer). Samples with $\approx 10 \mathrm{mg}$ were loaded into standard aluminum DSC pans. The samples were heated to $120^{\circ} \mathrm{C}$ at $10^{\circ} \mathrm{C} / \mathrm{min}$, held isothermally for $5 \mathrm{~min}$ to undo the thermal history, and cooled to $-50^{\circ} \mathrm{C}$ at $10^{\circ} \mathrm{C} / \mathrm{min}^{-1}$. Then samples were heated again to $120^{\circ} \mathrm{C}$ to obtain the data. Nematic-isotropic transition was identified as the local minimum of the exothermic peak on heating. The existence of the glass transition was estimated from the drastic change of the slope at lower temperature range. 
Mechanical analysis. The stress-strain curves for LCE films at two typical phases, nematic at $20^{\circ} \mathrm{C}$ and isotropic at $80^{\circ} \mathrm{C}$, in tensile mode, were obtained using a commercial instrument (EMX1000, IMADA) under temperature control of sample environment. The resulting sample width, thickness and effective length were, $10 \mathrm{~mm}, 0.2 \mathrm{~mm}$, and $30 \mathrm{~mm}$, respectively. The strain was increased at different rates of extension of $0.00042,0.0042,0.042$ and $0.42 \mathrm{~s}^{-1}$ to evaluate the viscoelastic response.

Adhesion tests. For measurement of adhesion, the probe-tack test was conducted. White light containing near infra-red light of a metal halide lamp (PCS-UMX350, NPI) was irradiated to the backside of the LCE film $\left(\sim 15 \times 15 \times 0.2 \mathrm{~mm}^{3}\right)$ fixed to a transparent glass slide with the thickness of $0.9 \mathrm{~mm}$ (Fig. 2a). The irradiated part was a circular spot with the diameter of $\sim 7 \mathrm{~mm}$. The light power $P$ was varied up to $2 \mathrm{~W} / \mathrm{cm}^{2}$ and was characterized using a power meter (model3664, HIOKI) at the wavelength of $780 \mathrm{~nm}$, around which the strong absorption band of ICG exists (Fig. 1b). On the frontside a flat surface of the glass probe (with the area $s 5 \times 5 \mathrm{~mm}^{2}$ ) with a certain weight $\left(w=100 \mathrm{~g}\right.$ ) was first placed to exert preload $F_{\mathrm{PL}} \sim 1 \mathrm{~N}$ and the state was held for a duel time, $t_{\mathrm{D}}=60 \mathrm{~s}$. Then, the probe was pulled at a certain speed, $v$, monitoring the force during the detachment (EMX1000, IMADA). For adhesion measurement under light irradiation $v=0.25$ $\mathrm{mm} / \mathrm{s}$. The maximum force at detachment was defined as adhesion forces $F_{\text {ad. }}$ Light irradiation was started $t_{\mathrm{L}}\left(=t_{\mathrm{D}}\right)$ before pulling. $T$ was monitored using a thermo camera (FLIR C2). Ambient temperature was $\sim 22^{\circ} \mathrm{C}$ and relative humidity was $\sim 40 \%$. Separately, temperature, $T$, dependent adhesion was also evaluated by controlling $T$ of the surroundings.

For demonstration of spatio-temporal control of adhesion, the $90^{\circ}$-peel test at constant load was performed. LCE strips with the width of $7 \mathrm{~mm}$, thickness of $0.2 \mathrm{~mm}$, and the length of $\sim 40 \mathrm{~mm}$ was first adhered to a transparent slide glass with the weight of $\sim 14 \mathrm{~g}$ hang at one end (Fig. 4a,b). 
By irradiating light at the peeling front, the rapid peeling was observed. Picking up polystyrene balls with the diameter of $5 \mathrm{~mm}$ and the weight of $0.2 \mathrm{~g}$ at room temperature and light-induced releasing of them were also demonstrated (Fig. 4d).

\section{ASSOCIATED CONTENT}

\section{Supporting Information.}

The Supporting Information is available free of charge at ,„,,,..

SI-Movie $1.90^{\circ}$-peel test under spatio-temporal light irradiation (MP4)

SI-Movie 2. Pick-and-release of plastic balls (MP4)

\section{AUTHOR INFORMATION}

\section{Corresponding Authors}

Takuya Ohzono - Research Institute for Electronics and Photonics, National Institute of Advanced Industrial Science and Technology (AIST) 1-1-1 Higashi, Tsukuba 305-8565, Japan. E-mail: ohzono-takuya@aist.go.jp

Eugene M. Terentjev - Cavendish Laboratory, University of Cambridge, J.J. Thomson Avenue, Cambridge, CB3 0HE, United Kingdom. E-mail: emt1000@cam.ac.uk

\section{Author Contributions}

EMT, TO and MOS designed the central concept of the present study. MOS designed the materials. TO, MOS and YN conducted experimental characterizations. The manuscript was 
written by TO and EMT through contributions of all authors. All authors have given approval to the final version of the manuscript.

\section{Funding Sources}

This work was supported by the European Research Council grant No: 786659.

\section{Notes}

The authors declare no competing financial interest.

\section{ACKNOWLEDGMENT}

T.O. gratefully acknowledge H Akiyama and S. Ito for useful discussions.

\section{REFERENCES}

(1) Benedek, I.; Feldstein, M. M. Handbook of Pressure-Sensitive Adhesives and Products: Fundamentals of Pressure Sensitivity, 1st ed.; CRC Press, 2008.

(2) Creton, C.; Ciccotti, M. Fracture and Adhesion of Soft Materials: A Review. Rep. Prog. Phys. 2016, 79, 046601.

(3) Kamperman, M.; Synytska, A. Switchable Adhesion by Chemical Functionality and Topography. J. Mater. Chem. 2012, 22, 19390-19401.

(4) Reddy, S.; Arzt, E.; Del Campo, A. Bioinspired Surfaces with Switchable Adhesion. Adv. Mater. 2007, 19, 3833-3837.

(5) Lin, P. C.; Vajpayee, S.; Jagota, A.; Hui, C. Y.; Yang, S. Mechanically Tunable Dry Adhesive from Wrinkled Elastomers. Soft Matter 2008, 4, 1830-1835. 
(6) Chan, E. P.; Smith, E. J.; Hayward, R. C.; Crosby, A. J. Surface Wrinkles for Smart Adhesion. Adv. Mater. 2008, 20, 711-716.

(7) Ohzono, T.; Teraoka, K. Switchable Bumps of a Bead-Embedded Elastomer Surface with Variable Adhesion. Soft Matter 2017, 13, 9082-9086.

(8) Liu, D.; Broer, D. J. Self-Assembled Dynamic 3D Fingerprints in Liquid-Crystal Coatings towards Controllable Friction and Adhesion. Angew. Chemie - Int. Ed. 2014, 126, 46304634.

(9) Heinzmann, C.; Coulibaly, S.; Roulin, A.; Fiore, G. L.; Weder, C. Light-Induced Bonding and Debonding with Supramolecular Adhesives. ACS Appl. Mater. Interfaces 2014, 6, $4713-4719$.

(10) Hohl, D. K.; Weder, C. (De)Bonding on Demand with Optically Switchable Adhesives. Adv. Opt. Mater. 2019, 7, 1-25.

(11) Zhou, Y.; Chen, M.; Ban, Q.; Zhang, Z.; Shuang, S.; Koynov, K.; Butt, H. J.; Kong, J.; Wu, S. Light-Switchable Polymer Adhesive Based on Photoinduced Reversible Solid-toLiquid Transitions. ACS Macro Lett. 2019, 8, 968-972.

(12) De Crevoisier, G.; Fabre, P.; Corpart, J. M.; Leibler, L. Switchable Tackiness and Wettability of a Liquid Crystalline Polymer. Science 1999, 285, 1246-1249.

(13) Warner, M., Terentjev, E. M. Liquid Crystal Elastomers; Oxford Univ. Press, 2007.

(14) Clarke, S. M.; Tajbakhsh, A. R.; Terentjev, E. M.; Remillat, C.; Tomlinson, G. R.; House, J. R. Soft Elasticity and Mechanical Damping in Liquid Crystalline Elastomers. J. Appl. 
Phys. 2001, 89, 6530-6535.

(15) Clarke, S. M.; Tajbakhsh, A. R.; Terentjev, E. M.; Warner, M. Anomalous Viscoelastic Response of Nematic Elastomers. Phys. Rev. Lett. 2001, 86, 4044-4047.

(16) Clarke, S. M.; Hotta, A.; Tajbakhsh, A. R.; Terentjev, E. M. Effect of Crosslinker Geometry on Equilibrium Thermal and Mechanical Properties of Nematic Elastomers. Phys. Rev. E 2001, 64, 8.

(17) Wang, T.; Lei, C. H.; Dalton, A. B.; Creton, C.; Lin, Y.; Fernando, K. A. S.; Sun, Y. P.; Manea, M.; Asua, J. M.; Keddie, J. L. Waterborne, Nanocomposite Pressure-Sensitive Adhesives with High Tack Energy, Optical Transparency, and Electrical Conductivity. Adv. Mater. 2006, 18, 2730-2734.

(18) Wang, T.; Colver, P. J.; Bon, S. A. F.; Keddie, J. L. Soft Polymer and Nano-Clay Supracolloidal Particles in Adhesives: Synergistic Effects on Mechanical Properties. Soft Matter 2009, 5, 3842-3849.

(19)

Ohzono, T.; Saed, M. O.; Terentjev, E. M. Enhanced Dynamic Adhesion in Nematic Liquid Crystal Elastomers. Adv. Mater. 2019, 31, 1902642.

(20) White, T. J.; Broer, D. J. Programmable and Adaptive Mechanics with Liquid Crystal Polymer Networks and Elastomers. Nat. Mater. Nature Publishing Group 2015, pp 10871098.

(21) Yu, Y.; Nakano, M.; Ikeda, T. Directed Bending of a Polymer Film by Light. Nature 2003, 425, 145-145. 
(22) Camacho-Lopez, M.; Finkelmann, H.; Palffy-Muhoray, P.; Shelley, M. Fast LiquidCrystal Elastomer Swims into the Dark. Nat. Mater. 2004, 3, 307-310.

(23) Liu, D.; Broer, D. J. New Insights into Photoactivated Volume Generation Boost Surface Morphing in Liquid Crystal Coatings. Nat. Commun. 2015, 6, 1-7.

(24) Marshall, J. E.; Ji, Y.; Torras, N.; Zinoviev, K.; Terentjev, E. M. Carbon-Nanotube Sensitized Nematic Elastomer Composites for IR-Visible Photo-Actuation. Soft Matter 2012, 8, 1570-1574.

(25) Yang, H.; Liu, J. J.; Wang, Z. F.; Guo, L. X.; Keller, P.; Lin, B. P.; Sun, Y.; Zhang, X. Q. Near-Infrared-Responsive Gold Nanorod/Liquid Crystalline Elastomer Composites Prepared by Sequential Thiol-Click Chemistry. Chem. Commun. 2015, 51, 12126-12129.

(26) Liu, W.; Guo, L.; Lin, B.; Zhang, X.; Sun, Y.; Yang, H. Near-Infrared Responsive Liquid Crystalline Elastomers Containing Photothermal Conjugated Polymers. Macromolecules 2016, 49, 4023-4030.

(27) Marshall, J. E.; Terentjev, E. M. Photo-Sensitivity of Dye-Doped Liquid Crystal Elastomers. Soft Matter 2013, 9, 8547-8551.

(28) Harvey, C. L. M.; Terentjev, E. M. Role of Polarization and Alignment in Photoactuation of Nematic Elastomers. Eur. Phys. J. E 2007, 23, 185-189.

(29) Squires, A. M.; Tajbakhsh, A. R.; Terentjev, E. M. Dynamic Shear Modulus of Isotropic Elastomers. Macromolecules 2004, 37, 1652-1659.

(30) Warner, M.; Bladon, P.; Terentjev, E. M. "Soft Elasticity" — Deformation without 
Resistance in Liquid Crystal Elastomers. J. Phys. II 1994, 4, 93-102.

(31) La Joie, E. N.; Barofsky, A. D.; Gregory, K. W.; Prahl, S. A. Patch Welding with a Pulsed Diode Laser and Indocyanine Green. Lasers Med. Sci. 1997, 12, 49-54.

(32) Saed, M. O.; Terentjev, E. M. Siloxane Crosslinks with Dynamic Bond Exchange Enable Shape Programming in Liquid-Crystalline Elastomers. Sci. Rep. 2020, 10, 6609.

(33) Saed, M. O.; Ambulo, C. P.; Kim, H.; De, R.; Raval, V.; Searles, K.; Siddiqui, D. A.; Cue, J. M. O.; Stefan, M. C.; Shankar, M. R.; Ware, T. H. Molecularly-Engineered, 4D-Printed Liquid Crystal Elastomer Actuators. Adv. Funct. Mater. 2019, 29, 1806412.

(34) Kotikian, A.; McMahan, C.; Davidson, E. C.; Muhammad, J. M.; Weeks, R. D.; Daraio, C.; Lewis, J. A. Untethered Soft Robotic Matter with Passive Control of Shape Morphing and Propulsion. Sci. Robot. 2019, 4, 1-11. 


\section{TOC content}

Light-controlled spatio-temporal dynamic adhesion is demonstrated exploiting the photothermal phase transition in a dye-doped nematic liquid crystal elastomer.
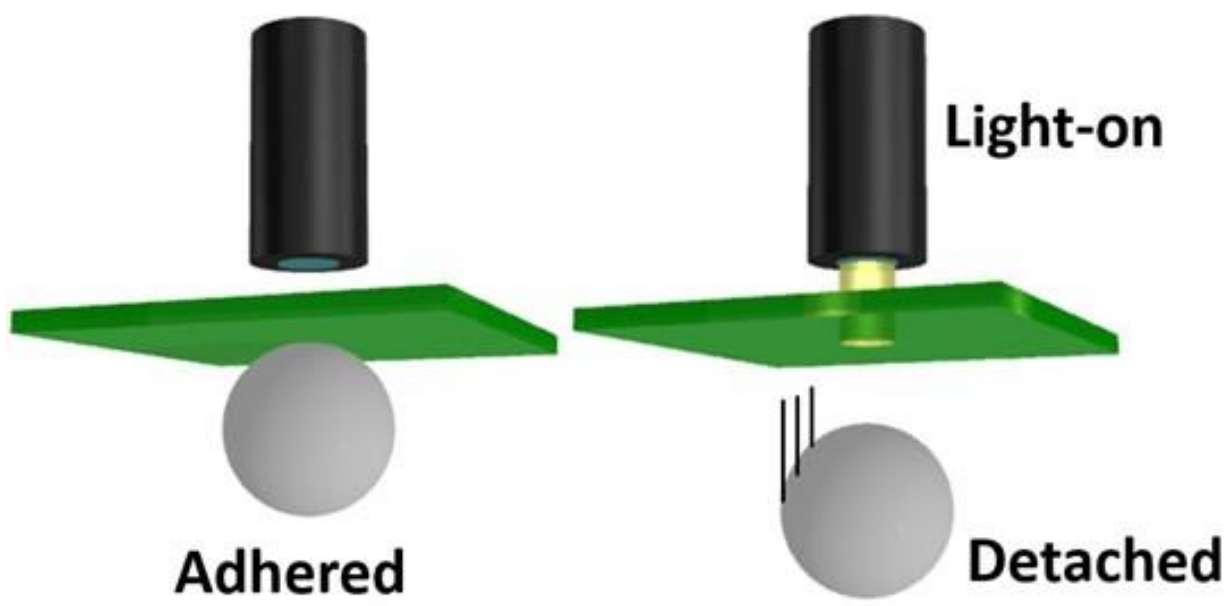